\title{
EDITORIAL
}

\section{The price of a pandemic}

\author{
With an ailing global economy, efforts to minimize virus spread and prevent loss of life \\ during a flu pandemic could be hindered by the fragile financial situation.
}

The potential dangers of a flu pandemic to society have long been appreciated. Given the ease of intercontinental travel and globalization of our economies, outbreaks on the scale of the 1918 Spanish flu pandemic, which infected up to $40 \%$ of the global population and claimed 50 million lives, would today have a devastating effect. In recent weeks, a new variant of an $\mathrm{H} 1 \mathrm{~N} 1$ influenza type A virus that normally causes a respiratory disease in pigs has quickly spread worldwide from its origins in Mexico. Although a number of swine flu-related fatalities have occurred, early signs indicate that most individuals infected have responded well to treatment with antiviral drugs.

Clearly, limiting loss of life by providing immediate access to antiviral drugs for those infected during a flu outbreak must be an urgent priority. However, aside from the immediate health issues, another early impact of such outbreaks is an economic one. Stock markets around the world reacted nervously to swine flu, with commodity markets and the travel industry suffering in particular. Despite assurances from health agencies around the world that meat products are safe and a lack of evidence to suggest that such viruses can be transmitted by eating infected meat, fears that global meat consumption would be severely affected led to decreases in the price of grain used for animal feed. Similarly, shares in travel companies and airlines also declined, while many such companies postponed or cancelled flights to outbreak areas. This was despite statements from WHO advising that restrictions on travel were not necessary. Conversely, shares in pharmaceutical companies that produce antiviral drugs and vaccines performed strongly.

Despite the immediate financial impact of scaling up responses to deal with the threat of a flu pandemic, the cost of inaction in terms of both human life and economic output could be far greater. As such, it is essential that clear communication between health care professionals and governments be maintained during an outbreak so that any actions taken are informed by the latest expert advice. The popular media has an important part to play in this process by keeping the public apprised of the facts, without exaggerating the potential threat from the virus. On the whole, the world media has covered the current flu outbreak in a responsible manner, but the temptation to scaremonger must continue to be resisted. This is more important now than ever, with social messaging services such as Twitter able to disseminate information quickly to millions of people. Increasing public anxiety levels further could lead to reactionary steps being taken, which place additional and unwarranted strain on an already fragile world economy, potentially leading to serious consequences for the quality of health care provision.

Investment in early warning systems, such as the European influenza surveillance system and the CDC surveillance system, together with coordinated strategies for responding to flu pandemics should ensure that countries such as the United States and members of the European Union are well placed to respond early and effectively to an outbreak, even in the current financial climate. However, the many other countries with economies that are already facing difficult times are likely to fare much worse. A coordinated international effort to provide aid to less developed countries that are facing the effects of a flu pandemic will therefore be of paramount importance.

In addition, providing financial support and scientific advice to less developed countries to improve health care facilities and outbreak detection systems will benefit the local population and improve the chances of avoiding a wider pandemic. Furthermore, careful analysis of the effectiveness of response mechanisms set into motion by the recent outbreak would help ensure that any future outbreak of a more virulent influenza strain would be quickly contained, reducing loss of life and limiting the global impact.

A clearer picture of the scale of the swine flu pandemic, and therefore the steps required to address the problem, should appear in the coming weeks. In the meantime, swine flu provides us with a timely reminder of the potential threat posed by more virulent influenza viruses and the importance of actively maintaining and improving our preparedness for future pandemics.

\section{FURTHER INFORMATION}

WHO: http://www.who.int/csr/disease/swineflu

CDC: http://www.cdc.gaov/swineflu/

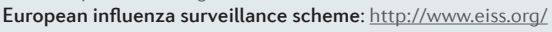

ALL LINKS ARE ACTIVE IN THE ONLINE PDF 